\title{
Bloch surface plasmon enhanced blue emission from InGaN/GaN light-emitting diode structures with Al-coated GaN nanorods
}

Guogang Zhang ${ }^{1,3}$, Zhe Zhuang ${ }^{1,3}, \mathrm{Xu} \mathrm{Guo}^{2,3}$, Fang-Fang Ren ${ }^{1,3,4,}$ a), Bin Liu ${ }^{1,3}$, Haixiong Ge ${ }^{2,3}$, Zili

$\mathrm{Xie}^{1,3}$, Ling Sun ${ }^{2,3}$, Ting Zhi ${ }^{1,3}$, Tao Tao ${ }^{1,3}, \mathrm{Yi} \mathrm{Li}^{1,3}$, Youdou Zheng ${ }^{1,3}$ and Rong Zhang ${ }^{1,3, a)}$

\author{
${ }^{1}$ Jiangsu Provincial Key Laboratory of Advanced Photonic and Electronic Materials, School of Electronic Science and \\ Engineering, Nanjing University, Nanjing 210093, P. R. China \\ ${ }^{2}$ College of Engineering and Applied Sciences, Nanjing University, Nanjing 210093, P. R. China \\ ${ }^{3}$ Nanjing National Laboratory of Microstructures, Nanjing University, Nanjing 210093, P. R. China \\ ${ }^{4}$ Department of Electronic Materials Engineering, Research School of Physics and Engineering, The Australian National \\ University, Canberra, ACT 0200, Australia
}

\begin{abstract}
InGaN/GaN light-emitting diode structures with Al-coated $\mathrm{GaN}$ nanorods were fabricated by using soft ultraviolet nanoimprint lithography. The intensity of light emission was found to be greatly enhanced due to the strong near-fields confined at the interface of $\mathrm{Al} / \mathrm{GaN}$ and extended to the multiple quantum wells (MQWs) active region. The dynamics of carrier recombination and the plasmon enhanced Raman scattering were also investigated, which provides a progressive view on the effective energy transfer between MQWs and surface plasmons.
\end{abstract}

Keywords: Bloch surface plasmons, light-emitting diodes, photoluminescence

a) Author to whom correspondence should be addressed. Electronic mails: ffren@nju.edu.cn and rzhang@,nju.edu.cn 


\section{Introduction}

Blue light emitting diodes (LEDs) based on III-nitride materials provide higher performance than other material systems [1] mainly due to their higher lumen efficiency, longer lifetime, and better stability. For the promising applications in full-color displays, printers, and solid-state lighting, an improvement of external quantum efficiency (EQE) that depends on the product of internal quantum efficiency (IQE) and light extraction efficiency (LEE) is essentially required. Thanks to the development of InGaN/GaN multiple quantum wells (MQWs) grown on a GaN epitaxial layer, the IQE of LEDs at room temperature has been greatly increased by improving material quality and suppressing the charge separation in quantum wells (quantum confined Stark effect, QCSE) [2,3]. However, the emission efficiency or EQE still mainly suffers from the limitation of LEE, which is greatly suppressed by total internal reflection resulted from the large refractive index contrast between $\mathrm{GaN}$ and air, and hence a low portion of light that can be extracted from the active region of LEDs [4].

To achieve the prospect of energy saving, numerous efforts related to the plasmonic effect have been made to improve the total output power [5-10]. Among these metallic micro/nano structures, nanoparticles (NPs) are of great interest and have demonstrated the ability in EQE enhancement via controlling the energy transfer between multiple quantum wells (MQWs) and localized surface plasmons (LSPs) [8]. However, these NPs are usually formed by the rapid thermal annealing of noble metals (e.g. $\mathrm{Au}, \mathrm{Ag}$, or $\mathrm{Pt}$ ) and the challenges lie in the accurate controlling of the shapes and dimension of these NPs. Therefore, the suppression of emission might be caused by the small size and low height due to the strong resonant absorption and poor light extraction as reported in [9]. Meanwhile, the metal NPs buried inside the LED for approaching the MQWs can even deteriorate the epitaxial quality of MQWs and consequently degrade the electrical characteristics of devices [6].

Two-dimensional metallic nano-structures with highly controlled dimensions have also been attempted to demonstrate plasmon-enhanced emission in LED structures based on the resonance coupling between the propagating Bloch surface plasmons (BSPs) and MQWs. As compared with self- 
organized NPs, highly controlled structures have the advantage of exciting long-range resonances, molding spectral/angular distribution of emission, and experimental reproducibility $[11,12]$. However, such a method still meets some limitations. For instance, the controllable nanostructure (e.g. nanocylinder) arrays are normally fabricated by electron-beam lithography (EBL) or focused ion beam (FIB) milling, which are much costly and complicated for LEDs. Moreover, for blue-light emission using $\mathrm{Au}$ or $\mathrm{Ag}$, the spacer layer between metal and MQWs is required to be extremely thin to ensure efficient SP coupling, which is impractical for device applications due to the ineffective p-doping of GaN and inferior ohmic contact [10].

In this work, we present the fabrication of InGaN/GaN LEDs with Al-coated GaN nanorods by using soft ultraviolet nanoimprint lithography [13], which offers lower cost and higher throughput as compared to EBL and FIB techniques $[14,15]$. The nontraditional plasmonic metal $\mathrm{Al}$ is chosen here due to its lower dissipation loss and deeper penetration depth rather than $\mathrm{Au}$ and $\mathrm{Ag}$ at blue-light spectral region $[16,17]$. Besides the desirable property in the blue and UV spectral regions, it also offers much lower cost and higher compatibility with III-nitrides. To avoid damaging the InGaN/GaN MQWs and also facilitate reciprocal vector for the excitation of BSPs via grating coupling, we create the nanorods array only within the $\mathrm{p}-\mathrm{GaN}$ layer. The presence of $\mathrm{Al}$ deposited on the bottom space outside nanorods is close to the MQW structure, which enables the excitation of BSP waves that converts the energy from non-radiative recombination to emitted photons. The consistency in theoretical simulation and experimental results suggested that the enhanced light emission of Al-coated LED structure with the reduced radiative recombination lifetime is attributed to the energy transfer from excitons in InGaN/GaN MQWs to BSPs by strong resonant coupling. The abnormal observations of blue-shift and enhancement of the longitudinal optical (LO) phonons in Al-coated structures indicated a resonant Raman scattering (RRS) process possibly assisted by metal-induced gap states at the $\mathrm{Al} / \mathrm{GaN}$ interfaces and surface plasmon resonance (SPR) effect. 


\section{Experimental methods}

The LED epitaxial structure for this study was grown by metal-organic chemical vapor deposition technique on the double-side polished c-plane sapphire substrates. To demonstrate the contribution of the nanorod array and Al coating for higher performance LEDs, we have fabricated four samples (A-D) by following the sequence of major fabrication steps in process flow. The as-grown structure (Sample A) consists of an unintentionally-doped GaN buffer, a $2 \mu \mathrm{m}$ thick n-GaN, a $220 \mathrm{~nm}$ thick active region with 15-pair InGaN/GaN MQWs, and a $590 \mathrm{~nm}$ thick p-GaN on the topmost surface. The peak emission of as-grown MQWs is centered at the wavelength of $475 \mathrm{~nm}$. The $\mathrm{SiO}_{2}$ mask for dry etching has been defined by using a nanoimprint lithography process [13] and it has a square lattice array with the dimension of $200 \mathrm{~nm}$ in depth, $200 \mathrm{~nm}$ in diameter, and $400 \mathrm{~nm}$ for pitch size. The Sample B was formed by etching down the LED wafer using $\mathrm{Cl}_{2} / \mathrm{BCl}_{3}$ ICP etching at $20{ }^{\circ} \mathrm{C}$ with $300 \mathrm{~W}$ ICP and 100 W RF. The depth of the etched nanorods was controlled at about $560 \mathrm{~nm}$. In order to remove the surface damages caused by dry etching and make the sidewall of nanorods smoother and straighter, the samples were wet etched in $3 \mathrm{~mol} / \mathrm{L} \mathrm{KOH}$ at $80^{\circ} \mathrm{C}$ for 25 minutes (named as Sample C). Finally, a $20 \mathrm{~nm}$ thick Al film was deposited onto the sample surface by high-vacuum e-beam evaporation to form Sample D.

The morphology of InGaN/GaN nanorods was measured by a JEOL JSM-7000F field emission scanning electron microscope (FE-SEM). The photoluminescence (PL) and time-resolved PL (TRPL) measurements have been performed excited by a $405 \mathrm{~nm}$ pulsed laser to investigate the effect of BSPs on the light emission property of LEDs. Both excitation and collection were carried out through the backside polished sapphire since the metal layer strongly attenuates the incident beams. The Raman scattering spectra were recorded in a backscattering configuration using the JYT6400 micro-Raman setup with a $514 \mathrm{~nm}$ laser as the excitation source, and the Raman shift was determined with accuracy better than $1 \mathrm{~cm}^{-1}$.

\section{Results and Discussion}


Figure 1(a) shows the schematic of the BSP-enhanced LED structure (Sample D). Due to the steep sidewall of nanorods, the Al film will be only coated on the top of $\mathrm{p}-\mathrm{GaN}$ nanorods and also the bottom space as shown in figure 1(a). The distance between the MQWs and the lower Al layer is approximately $20 \mathrm{~nm}$. Figures 1(b) and (c) show the top view and cross-sectional SEM images of the nanorod structures. The as-etched nanorod has a profile of conical frustum with a height of $560 \mathrm{~nm}$. After dipping the sample into $\mathrm{KOH}$ solution for 25 minutes, the average diameter of nanorods was found to be considerably reduced from $260 \mathrm{~nm}$ (as-etched) to $190 \mathrm{~nm}$, and the cross-section shape was changed from trapezoid into straight as illuminated in figures 1(d) and (e). The underlying mechanism for removing the etch-damaged region and obtaining the vertical shape with $\mathrm{KOH}$ treatment is identified as the different etching rates between $\mathrm{N}$-face and Ga-face [18].

Figure 2 shows the time-integrated PL spectra of the Samples A (as-grown LED structure), B (asetched LED structure), C (KOH-treated LED structure), and D (Al-BSP LED structure) at room temperature, respectively. The peak positions of light emission from the InGaN/GaN active layer in Samples A-D were observed at 476, 475, 474, and $470 \mathrm{~nm}$, respectively. For c-plane LED structures, it is well known that piezoelectric polarization field is present in $\mathrm{InGaN} / \mathrm{GaN}$ quantum well structures, which will cause the spatial separation of electron and holes, thus leading to the red-shift of quantum well emission and degrading the recombination efficiency, as called QCSE [19,20]. Such effect will be suppressed as compressive strain is released in quantum well structures. In this work, the strain involved in InGaN/GaN MQWs is partially released after etching from continuous epilayer into nanorod structures. Thus, compared with Samples A and B, the light emission exhibits a slight blue-shift in wavelength and enhancement in intensity. The comparison of Samples B and C suggests that the further reduction of piezoelectric field occurs in MQWs for nanorods with smaller size due to the relaxation of compressive strain. As compared to Sample A, the PL intensities from Samples B and C have a slight enhancement mainly due to the combined contribution of partial relaxation of piezoelectric field and surface morphology. Although the output could be enhanced by modulating the optical modes via 
photonic crystal patterns in Samples B and C, most of the emitted light leaks frontward to the free space rather than to the substrate, which can be confirmed based on the analysis of the dispersion diagram of electromagnetic waves in the photonic crystal regions (not shown). We therefore infer that in Samples B and $\mathrm{C}$, the surface morphology may have slight influence on the measured PL intensity, since the emissions were collected from the backside of samples. Surprisingly, a 2.6 times of PL enhancement in Sample D as compared to Sample C was observed, which is higher than that would be expected from reflection mechanism alone [5]. As shown in the inset of figure 2, the PL enhancement of Sample D relative to Sample C is dominantly centered at $467 \mathrm{~nm}$, which is exactly the wavelength of BSP mode excited on the interface of lower Al layer and p-GaN. It is therefore believed that the higher PL intensity in Sample D is due to the increase of spontaneous emission rate and internal quantum efficiency via Al BSP-coupling.

To testify the PL measurement results and understand the physical mechanism behind, we calculated the electric field intensity enhancement spectrum of Sample D by detecting $\left|E_{\mathrm{z}}\right|^{2} /\left|E_{\mathrm{z} 0}\right|^{2}$ at the interface of the lower $\mathrm{Al}$ nanostructures and $\mathrm{p}-\mathrm{GaN}$ layer based on finite-difference time-domain (FDTD) method. Here $\left|E_{z 0}\right|^{2}$ is the field intensity of incident light source located in the MQWs. The maximum electric field enhancement occurs at a free-space wavelength of $467 \mathrm{~nm}$. In figures 3(b) and (c), we plotted out the electric field distribution in Samples C and D at the wavelength of $467 \mathrm{~nm}$ based on the rigorous coupled wave analysis (RCWA). It can be found that the corresponding electric fields in Sample D are strongly confined near the lower surface of Al nanostructures and exponentially decay into the InGaN/GaN MQWs due to the excitation of BSP mode, therefore, the electric field intensity in Sample $\mathrm{C}$ without $\mathrm{Al}$ nanostructures is relatively weak. The large field enhancements occur at wavelengths within the MQW emission line shape, thereby increasing the QW radiation efficiency and output of PL intensity. The minor wavelength difference between the measured PL peak and the simulated BSP mode is acceptable by consideration of the errors in fabrication and simulation. 
To further demonstrate the plasmonic effects on improving the performance of the MQW LEDs, we carry out the TRPL measurements on all samples with a time-correlated single photon counting (TCSPC) setup at a temperature of $20 \mathrm{~K}$ (see figure 4). In our case, the TRPL traces could be fitted to a single exponential function [21] due to the frozen up of the non-radiative recombination centers at the low temperature of $20 \mathrm{~K}$. By directly fitting results, the spontaneous emission lifetimes $\left(\tau_{\mathrm{PL}}\right)$ for Samples A-D can be obtained as 20.04, 17.64, 17.49, and $14.13 \mathrm{ns,}$, respectively. The reduction lifetime of Samples B and C in contrast with Sample A is the result of increased wavefunction overlap of electrons and holes in the InGaN/GaN MQWs with the partially reduced piezoelectric field. While the faster decay time of Al-BSP LEDs can be attributed to resonance coupling between MQWs and BSPs. When the energy of the excitons in MQW is close to the electron vibrational energy of the LSP in the metal NPs, the recombination rate of excitons can be increased via fast recombination path due to the energy transfer from excitons to BSPs.

Figure 5 shows the normalized Raman scattering spectra collected from all the samples in $Z(-,-) \bar{Z}$ geometry, respectively, where "_," denotes a nonpolarized condition. The prominent peak at $569 \mathrm{~cm}^{-1}$ is assigned to the $\mathrm{E}_{2}^{\mathrm{h}}$ mode of $\mathrm{GaN}$, and the peaks around $734 \mathrm{~cm}^{-1}$ are assigned to the $\mathrm{A}_{1}$ (LO) phonon mode of GaN [22]. We also observed an additional peak around $700 \mathrm{~cm}^{-1}$ in Samples B, C, and D, which shifts toward the lower frequency direction with decreasing diameter of nanorods. This additional vibrational mode has been reported in similar GaN nanowire structures and regarded as surface optical (SO) phonon mode [23]. An abnormal observation is that the Raman modes of $\mathrm{A}_{1}$ (LO) and SO phonon modes in Sample D exhibit nearly 3 times of enhancement at 740 and $700 \mathrm{~cm}^{-1}$, respectively, which is an indication of strong interaction between photons and BSP modes occurring at the $\mathrm{Al} / \mathrm{GaN}$ interface [22]. In addition, a blue shift of the $\mathrm{SO}$ and $\mathrm{A}_{1}(\mathrm{LO})$ modes were also observed accompanied with a broader spectral linewidth in Sample D, which is attributed to the higher carrier concentration achieved after the deposition of $\mathrm{Al}$ nanostructures [22]. 
To quantify the difference of carrier concentration with or without Al deposition, we theoretically fit the measured Raman intensity of the LO phonon-plasmon coupled $\left(\mathrm{L}^{+}\right)$mode by employing the following expression $[22,24]$

$$
I(\omega)=S A(\omega) \operatorname{Im}\left[-\frac{1}{\varepsilon(\omega)}\right]
$$

here $\omega$ is the Raman frequency, $S$ a proportionality constant, and $\varepsilon(\omega)$ the dielectric function. The parameter $A(\omega)$ is given by

$$
\begin{aligned}
A(\omega)= & 1+2 C \omega_{T}^{2}\left[\omega_{P}^{2} \gamma\left(\omega_{T}^{2}-\omega^{2}\right)-\omega^{2} \Gamma\left(\omega^{2}+\gamma^{2}-\omega_{P}^{2}\right)\right] / \Delta \\
& \left.+\left(C^{2} \omega_{T}^{4} / \Delta\right)\left\{\omega_{P}^{2}\left[\gamma\left(\omega_{L}^{2}-\omega_{T}^{2}\right)\right]+\Gamma\left(\omega_{P}^{2}-2 \omega^{2}\right)\right]+\omega^{2} \Gamma\left(\omega^{2}+\gamma^{2}\right)\right\} /\left(\omega_{L}^{2}-\omega_{T}^{2}\right)
\end{aligned}
$$

where

$$
\Delta=\omega_{P}^{2} \gamma\left[\left(\omega_{T}^{2}-\omega^{2}\right)^{2}+(\omega \Gamma)^{2}\right]+\omega^{2} \Gamma\left(\omega_{L}^{2}-\omega_{T}^{2}\right)\left(\omega^{2}-\gamma^{2}\right)
$$

In Eqs. (2) and (3), $\omega_{T}, \omega_{L}$, and $\omega_{P}$ represent the unstrained TO, LO phonon and plasmon frequencies, $\gamma$ and $\Gamma$ the plasmon and phonon damping constants, and $C$ the Faust-Henry coefficient for hexagonal GaN. The dielectric function $\varepsilon(\omega)$ is given by a sum of the contribution of plasmons and phonons

$$
\varepsilon(\omega)=\varepsilon_{\infty}\left[1+\left(\omega_{L}^{2}-\omega_{T}^{2}\right) /\left(\omega_{T}^{2}-\omega^{2}-\mathrm{i} \omega \Gamma\right)-\omega_{P}^{2} /\left(\omega^{2}+\mathrm{i} \omega \gamma\right)\right]
$$

The strain free values of $\omega_{T}=533 \mathrm{~cm}^{-1}$ and the un-coupled $\omega_{L}=735 \mathrm{~cm}^{-1}$ are used as constants for fitting. The profile of the experimental data and the theoretical fitted curves of phonon-plasmon coupled $\mathrm{L}^{+}$modes are consistent very well as shown in the inset of figure 5. The fitting parameters $\gamma, \Gamma$, and $\omega_{P}$ can be extracted from the above line shape analysis. They are related to the free-carrier concentration $(n)$ and effective electron mass $\left(m^{*}=0.2 m_{e}\right)$ by

$$
\omega_{P}^{2}=\frac{4 \pi n e^{2}}{\varepsilon_{\infty} m^{*}}
$$

where $\varepsilon_{\infty}$ is the high frequency dielectric constant. As a result, the carrier concentration can be determined to be $2.7 \times 10^{17}$ and $1.6 \times 10^{17} \mathrm{~cm}^{-3}$ for the case with and without $\mathrm{Al}$ nanostructures, 
respectively. The higher electron concentration in Sample D may provide screening effect on the piezoelectric field, which also contributes to the blue-shift of light emission as described above.

We notice that the photon energy of the $514 \mathrm{~nm}$ laser $(2.41 \mathrm{eV})$ is much lower than the bandgap of $\mathrm{GaN}(3.4 \mathrm{eV})$ and therefore few free electrons can be excited from valence band into conduction band of GaN directly. However, the higher electron concentration can be generated from the sub-band electronic states, e.g., metal-induced gap states (MIGS) formed at the metal/GaN interfaces $[25,26]$. Noted that the Schottky barrier is $1.5 \mathrm{eV}$ for the $\mathrm{Al} / \mathrm{GaN}$ interface [25], the electrons in MIGS state can be promoted and resonate with unoccupied electronic states in the conduction bands of $\mathrm{GaN}$ [26]. The emission of a Raman photon will consequently occur through the scattering of excited electron to another state and then the recombination of electrons with the hole left in the gap states. As expected, the interface of AlBSP in Sample D contains more electrons rather than the other samples due to the electron transfer from MIGS states to GaN, which finally results in the broadening and high frequency shift of SO and LO modes.

\section{Conclusions}

In this study, an InGaN/GaN MQW LED structure with Al-coated GaN nanorods has been demonstrated to exhibit improved performance and relatively high efficiency. The low cost Al metal exhibits superior performance in blue spectral region and the great enhancement of light emission is due to the strong confinement of optical energy in the active region of InGaN/GaN MQWs. The phenomena of faster radiative recombination rate and enhanced Raman scattering are also observed and discussed. Our findings provide the foundation for rapid development of high-efficiency and low-cost LED devices.

\section{Acknowledgments}

This work was supported by Special Funds for Major State Basic Research Project (Nos. 2011CB301900 and 2012CB619304), Hi-tech Research Project (No. 2014AA032605), National Nature 
Science Foundation of China (Nos. 11104130, 61274003, 60990311, 61176063, and 61422401), Program for New Century Excellent Talents in University (No. NCET-11-0229), Nature Science Foundation of Jiangsu Province (Nos. BK2011556, BK2011010, BK2010385, BY2013077 and BE2011132), Funds of Key Laboratory (No. 9140C140102120C14), Scientific Innovation Research of College Graduate in Jiangsu Province (CXZZ12_0052), PAPD, the Fundamental Research Funds for the Central Universities, the Research Funds from NJU-Yangzhou Institute of Opto-electronics, and the Australian Research Council Discovery Early Career Researcher Award (DE130101700). 


\section{REFERENCES}

[1]. J. K. Kim, T. Gessmann, H. Luo and E. F. Schubert 2004 Appl. Phys. Lett. 844508

[2]. S. Nakamura and S. F. Chichibu 2000 Introduction to Nitride Semiconductor Blue Lasers and Light Emitting Diodes (Taylor \& Francis)

[3]. N. Tansu, H. Zhao, G. Liu, X. H. Li, J. Zhang, H. Tong and Y. K. Ee 2010 IEEE Photonics J. 2241

[4]. S. F. Chichibu, A. Uedono, T. Onuma, B. A. Haskell, A. Chakraborty, T. Koyama, P. T. Fini, S. Keller, S. P. Denbaars, J. S. Speck, U. K. Mishra, S. Nakamura, S. Yamaguchi, S. Kamiyama, H. Amano, I. Akasaki, J. Han and T. Sota 2006 Nat. Mater. 5810

[5]. K. Okamoto, I. Niki, A. Shvartser, Y. Narukawa, T. Mukai and A. Scherer 2004 Nat. Mater. 3601

[6]. M. K. Kwon, J. Y. Kim, B. H. Kim, I. K. Park, C. Y. Cho, C. C. Byeon and S. J. Park 2008 Adv. Mater. 20 1253

[7]. T. S. Oh, H. Jeong, Y. S. Lee, J. D. Kim, T. H. Seo, H. Kim, A. H. Park, K. J. Lee and E. K. Suh 2009 Appl. Phys. Lett. 95111112

[8]. C. W. Huang, H. Y. Tseng, C. Y. Chen, C. H. Liao, C. Hsieh, K. Y. Chen, H. Y. Lin, H. S. Chen, Y. L. Jung, Y. W. Kiang and C. C. Yang 2011 Nanotechnology 22475201

[9]. S. Jiang, Z. Hu, Z. Z. Chen, X. X. Fu, X. Z. Jiang, Q. Q. Jiao, T. J. Yu and G. Y. Zhang 2013 Opt. Express 21 12100

[10]. C. H. Lu, C. C. Lan, Y. L. Lai, Y. L. Li and C. P. Liu 2011 Adv. Funct. Mater. 214719

[11]. A. Fadil, D. Iida, Y. T. Chen, J. Ma, Y. Y. Ou, P. M. Petersen and H. Y. Ou 2014 Sci. Rep. 46392

[12]. G. Lozano, D. J. Louwers, Said R. K. Rodriguez, S. Murai, Olaf T. A Jansen, M. A. Verschuuren and J. G. Rivas 2013 Light-Sci. Appl. 2 e66

[13]. Z. Zhuang, X. Guo, G. G. Zhang, B. Liu, R. Zhang, T. Zhi, T. Tao, H. X. Ge, F. F. Ren, Z. L. Xie and Y. D. Zheng 2013 Nanotechnology 24405303

[14]. J. J. Wierer, Jr., A. David and M. M. Megens 2009 Nat. Photonics 3163

[15]. P. Yu, C. H. Chiu, Y.-R. Wu, H. H. Yen, J. R. Chen, C. C. Kao, H. W. Yang, H. C. Kuo, T. C. Lu, W. Y. Yeh and S. C. Wang 2008 Appl. Phys. Lett. 93081110

[16]. K. Okamoto, I. Niki, A. Shvartser, Y. Narukawa, T. Mukai and A. Scherer 2004 Nat. Mater. 3601

[17]. M. W. Knight, N. S. King, L. Liu, H. O. Everitt, P. Nordlander and N. J. Halas 2014 ACS Nano 8834

[18]. S. Y. Bae, D. J. Kong, J. Y. Lee, D. J. Seo and D. S. Lee 2013 Opt. Express 2116854

[19]. Q. Wang, J. Bai, Y. P. Gong and T. Wang 2011 J. Phys. D Appl. Phys. 44395102 
[20]. Q. Li, K. R. Westlake, M. H. Crawford, S. R. Lee, D. D. Koleske, J. J. Figiel, K. C. Cross, S. Fathololoumi, Z. T. Mi and G. T. Wang 2011 Opt. Express 1925528

[21]. K. Okamoto, I. Niki, A. Scherer, Y. Narukawa, T. Mukai and Y. Kawakami 2005 Appl. Phys. Lett. 87 071102

[22]. H. Harima 2002 J. Phys.: Condens. Matter 14 R967

[23]. J. H. Zhu, J. Q. Ning, C. C. Zheng, S. J. Xu, S. M. Zhang and H. Yang 2011 Appl. Phys. Lett. 99113115

[24]. K. Jeganathan, R. K. Debnath, R. Meijers, T. Stoica, R. Calarco, D. Grützmacher and H. Lüth 2009 J. Appl. Phys. 105123707

[25]. S. Picozzi, A. Continenza, G. Satta, S. Massidda and A. J. Freeman 2000 Phys. Rev. B 6116736

[26]. C. Y. Liu, M. M. Dvoynenko, M. Y. Lai, T. H. Chan, Y. R. Lee, J.-K. Wang and Y. L. Wang 2010 Appl. Phys. Lett. 96033109 


\section{FIGURE CAPTIONS}

Figure 1. (Color online) (a) Schematics of the fabrication of Sample D. (b) The top-view and (c) cross-sectional SEM images of Sample B. (d) The top-view and (e) cross-sectional SEM images of Sample C.

Figure 2. (Color online) Room-temperature PL spectra of Samples A-D. The inset shows the PL enhancement of Sample D relative to Sample C.

Figure 3. (Color online) (a) The simulated electric field intensity $\left(\left|E_{\mathrm{z}}\right|^{2} /\left|E_{\mathrm{z} 0}\right|^{2}\right)$ enhancement spectrum in Sample D. $E_{\mathrm{z}}$-field intensity distribution in (b) Sample C and (c) Sample D at the wavelength of $467 \mathrm{~nm}$.

Figure 4. (Color online) Time-resolved photoluminescence decay curves from Samples A (black), B (green), C (blue), and D (red) at $20 \mathrm{~K}$.

Figure 5. (Color online) Raman scattering spectra of Samples A-D recorded in $Z(-,-) \bar{Z}$ geometry at room temperature. The inset shows the experimental data and the theoretical fitted curves of $\mathrm{L}^{+}$modes for Samples C and D. 


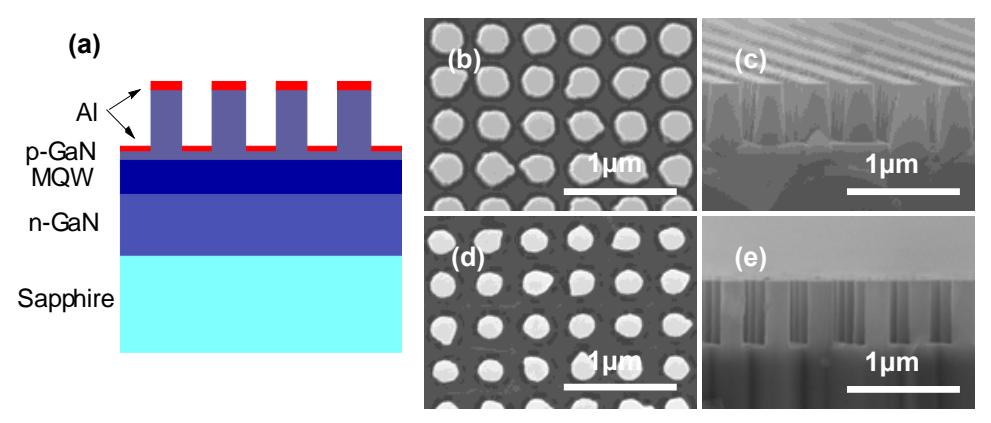

Figure1 By Guogang Zhang et al. 


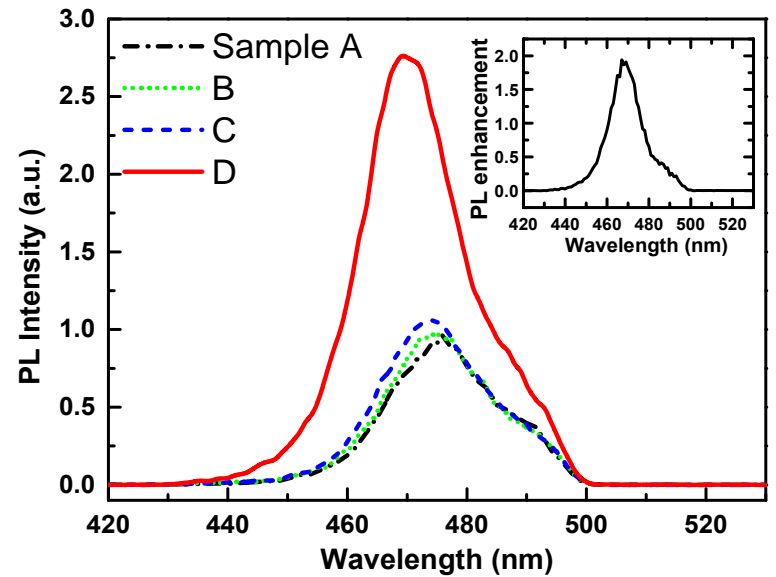

Figure 2 By Guogang Zhang et al. 

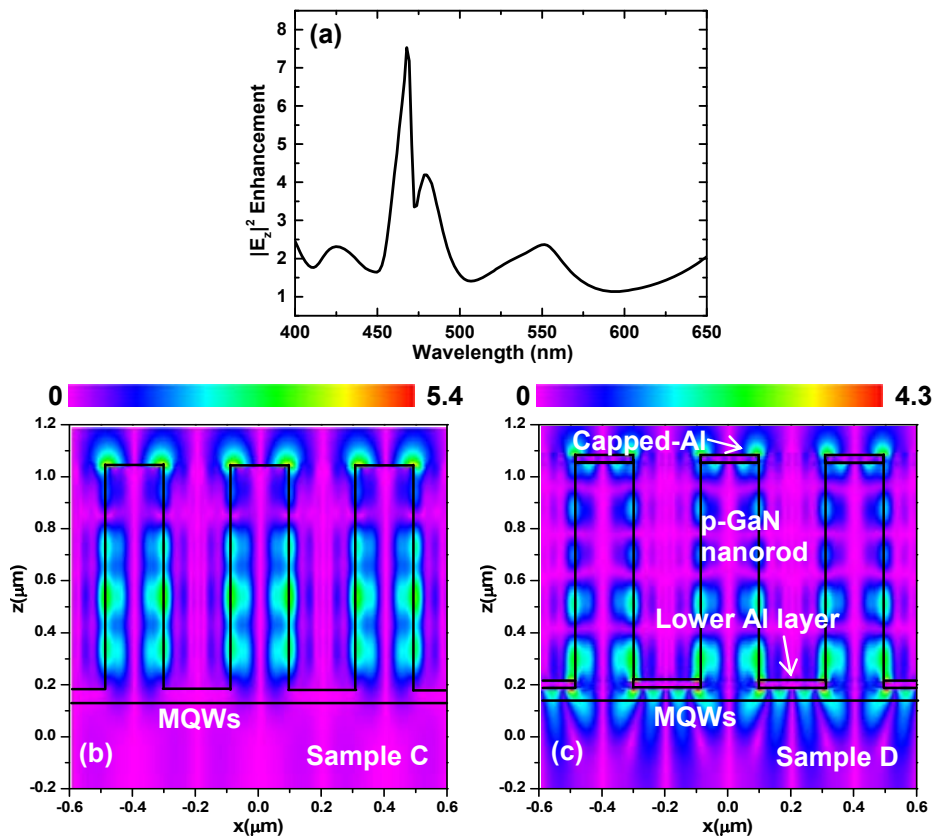

Figure 3 By Guogang Zhang et al. 


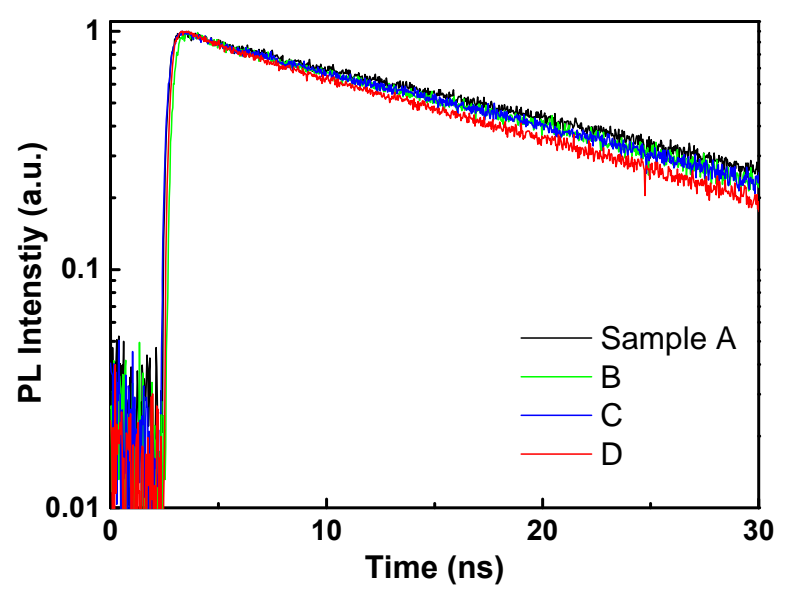

Figure 4 By Guogang Zhang et al. 


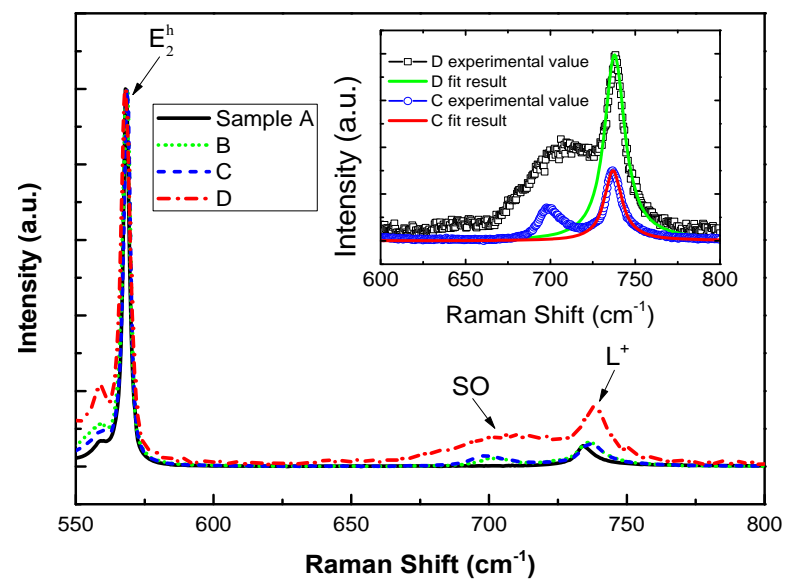

Figure 5 By Guogang Zhang et al. 